\title{
CISTOS DE ECHINOCOCCUS VOGELI EM FÍGADO DE PACA (CUNICULUS PACA) ORIGINÁRIA DO ESTADO DO ACRE, BRASIL
}

\author{
Ulysses G. Meneghelli, Ana L. C. Martinelli e Maria A. S. Llorach Velludo
}

\begin{abstract}
Quatro dos pacientes com doença hidática policística por nós observados referiam ter reconhecido doença no figado de pacas cacadas a fim de serem utilizadas como alimento; as vísceras desses animais eram, habitualmente, dadas aos câes domésticos. Todos os nossos 7 pacientes referiam contactos com cães que previamente haviam ingerido vísceras de pacas. $O$ exame de figado considerado doente por um dos pacientes e retirado de uma paca abatida na mesma região (Estado do Acre, Brasil) de onde provieram os casos humanos mostrou a presença de cistos hidáticos. As características dos acúleos do protoscolex indicaram tratar-se da forma larval do Echinococcus vogeli. Essas observaçôes confirmam a participação da paca no ciclo biológico do E. vogeli e a via pela qual o homem pode tornar-se o hospedeiro intermediário alternativo desse equinococo.
\end{abstract}

Palavras-chaves: Equinococose. Doença hidática policistica. Echinococcus vogeli.

Casos de hidatidose policística têm sido descritos nos paises amazônicos vizinhos do Brasil $^{2} \mathrm{e}$ também na Amazônia brasileira ${ }^{8} 9$. O agente etiológico dessa doença é o Echinococcus vogel ${ }^{2} 11$ que tem como hospedeiro intermediário principal a paca (Cuniculus paca). Fora do território brasileiro, cistos de $E$. vogeli já foram identificados em pacas capturadas na Colômbia ${ }^{3}$ e na Bolivia 5 . O homem, hospedeiro intermediário alternativo, adquire a doença contaminando-se por via oral com ovos emitidos nas fezes de cães domésticos ou de caça que previamente tenham ingerido vísceras de pacas portadoras da doença.

Todos os sete pacientes com hidatidose policistica que estudamos 89 , eram provenientes do Estado do Acre, e referiam contato com cães que freqüentemente se alimentavam com visceras de pacas; 4 desses pacientes já haviam identificado pacas com doença no figado, com caracteristicas que julgamos altamente sugestivas de hidatidose. Com a finalidade de comprovar a doença hidática nesses hospedeiros intermediários, instruímos alguns de nossos pacientes

Departamentos de Clínica Médica e de Patologia. Faculdade de Medicina de Ribeirão Preto, Universidade de São Paulo, Ribeirão Preto, SP.

Endereço para correspondência: Dr. Ulysses G. Meneghelli. Disc. de Gastroenterologia, Depto de Clínica Médica, Hospital das Clínicas, FMRP/USP. 14048 Ribeirão Preto, SP.

Recebido para publicação em: 31/07/90. provenientes do Acre para que nos enviassem materiais suspeitos colhidos de pacas caçadas em suas regiões.

\section{MATERIAL E MÉTODOS}

Em 1989, recebemos um figado de paca abatida por caçadores no Município de Sena Madureira, Estado do Acre, fixado em formol-tamponado e trazido, em mãos, ao Hospital das Clínicas de Ribeirão Preto.

Após exame macroscópico, o material foi submetido a processamento para exame histológico. Obtiveram-se cortes de $5 \mu \mathrm{m}$ corados pela hematoxilina-eosina (HE) que foram examinados ao microscópio óptico para a identificação da natureza dos cistos. $\mathrm{Na}$ tentativa de obter elementos para a definição da espécie do Echinococcus foram feitas medidas do comprimento total dos acúleos encontrados em boas condições e de suas duas partes: cabo e lâmina.

\section{RESULTADOS}

O exame macroscópico do fígado (Figura 1) evidenciou vários cistos subcapsulares de paredes delgadas. $O$ diàmetro variou de 0,2 a $1,2 \mathrm{~cm}$, alguns rotos e vazios e outros contendo escassa quantidade de líquido levemente leitoso.

Ao exame histológico observaram-se cistos hidáticos com membrana cuticular externa multilamelar e amorfa e membrana germinativa com epitélio sincicial delgado, com extensas áreas de degeneração, ocasionalmente constituindo cápsula prolígera. No 


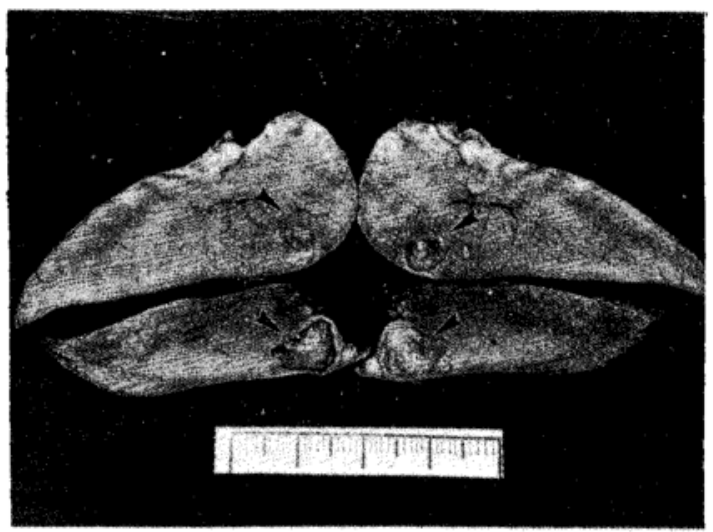

Figura 1 - Secções de figado de paca onde podem ser observados cistos hidaticos subcapsulares (setas).

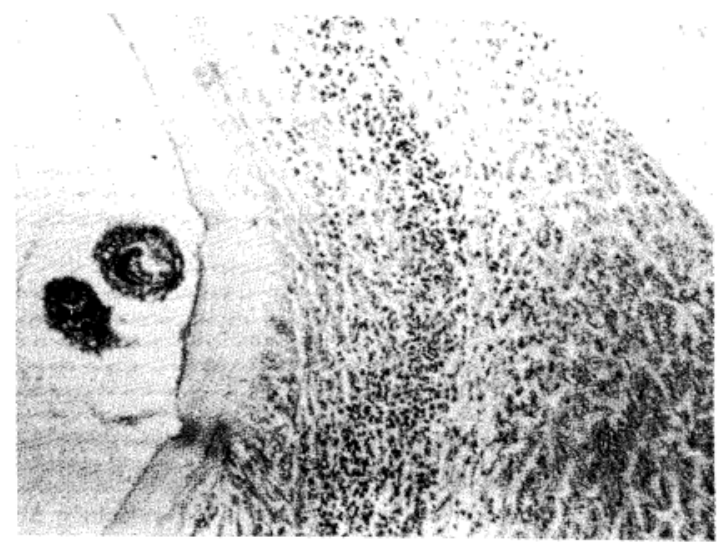

Figura 2 - Cisto hidático em parênquina hepático de paca. Processo inflamatório crônico permeando tecido fibroso que envolve camada amorfa multilamelar. Observa-seo epitélio germinativo e cápsula prolígera contendo dois protoscólices de $\mathrm{E}$. vogeli. (HE, 120x).

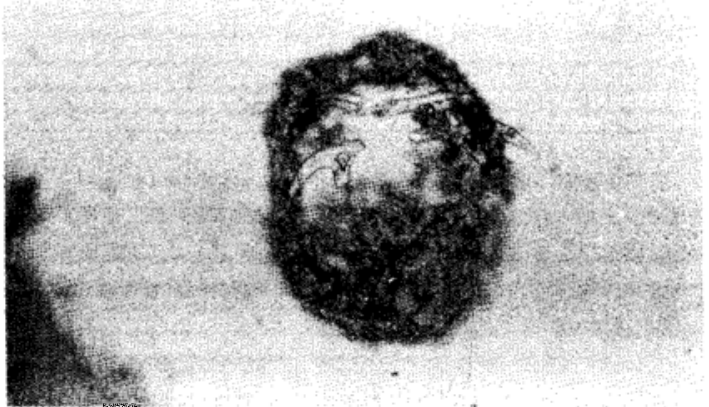

Figura 3-Escólex degenerado com acúleos grandes, soltos, com forma e dimensões dentro dos padrões descritos para $o$ E. vogeli. $(H E, 600 x)$. interior dessas cápsulas foram identificados protoscólices de equinococos com alterações autoliticas, o que não impediu de serem reconhecidos como maduros. A membrana cuticular às vezes invaginava-se na luz assumindo aspecto cerebriforme. $\mathrm{Na}$ luz dos cistos encontraram-se, também, detritos amorfos, microconcreções calcáreas e acúleos soltos, em geral fragmentados. Os cistos eram envoltos por cápsula fibrosa densa com infiltrado inflamatório mononuclear que os separavam do parênquima hepático adjacente (Figura 2).

Os acúleos observados apresentavam-se com convexidade harmoniosa, arco laminar bem pronunciado e a lâmina perceptivelmente maior que o cabo, como pode ser visto na Figura 3. Em três dos vários acúleos observados foi possivel a determinação de suas dimensões, que são apresentadas na Tabela 1.

Tabela 1 - Medidas efetuadas em três acúleos integros.

\begin{tabular}{|c|c|c|c|c|}
\hline \multirow{2}{*}{ Acúleo } & \multicolumn{3}{|c|}{ Extensão $(\mu \mathrm{m})$} & \multirow{2}{*}{$\frac{\%}{\text { Cabo - Lâmina }}$} \\
\hline & Total & Cabo & Lâmina & \\
\hline 1 & 40,0 & 13,8 & 26,2 & $34,5-65,5$ \\
\hline 2 & 39,3 & 13,4 & 25,9 & $34,1-65,9$ \\
\hline 3 & 41,0 & 14,7 & 26,3 & $34,9-65,1$ \\
\hline
\end{tabular}

A forma e as dimensões dos acúleos permitiram a conclusão de que os cistos hidáticos observados no figado da paca estudada pertenciam à espécie $E$. vogeli ${ }^{13}$.

\section{DISCUSSÃO}

Há antigos relatos do encontro de cistos hidáticos em roedores silvestres do interior do Brasil. Lutz $^{6}$, em cutia caçada em Jacutinga, perto de Bauru (SP), em 1907, encontrou "tanto nos pulmões, como no figado e no baço, grande aglomeração de pequenas vesículas hialinas" identificadas como a forma larval de um Echinococcus. Brumpt e Joyeux ${ }^{1}$, em 1924, encontraram 3 pequenos cistos hidáticos no figado $\mathrm{e}$ um no baço de uma cutia capturada em Albuquerque Lins (hoje, Lins, SP); o parasita foi descrito como uma nova espécie que recebeu a designação Echinococcus cruzi. Há evidências de que esse parasita não seja outro senão o $E$. oligarthrus 12 .

Mais recentemente, encontraram-se cistos de $E$. oligarthrus em cutias procedentes das proximidades de Belém, PA, e cistos de $E$. vogeli em pacas de Serra do Navio, território do Amapá 3 . 
No presente trabalho foram identificados cistos hidáticos em paca capturada em Sena Madureira, Estado do Acre. A forma dos acúleos, a maior proporção das dimensões da lâmina em relação ao cabo e seu tamanho total, maior do que de outras espécies de Echinococcus, caracterizam a forma larval do $E$. vogeli ${ }^{13}$.

Comprova-se, portanto, a presença do $E$. vogelis em paca originária da mesma região de onde provieram os casos humanos por nós observados 89 . Esse fato, ao lado das informações dos pacientes de que tiveram contactos com cães que haviam ingerido vísceras de pacas e de que haviam identificado lesões hepáticas sugestivas dos cistos parasitários nesses animais, confirma que esse roedor silvestre participa do ciclo biológico do $E$. vogeli e indica a via pela qual o homem pode tornar-se o hospedeiro intermediário alternativo desse equinococo.

A ocorrência da doença dentro e fora da área amazônica4 71014 indica que, embora pouco freqüente, essa hidatidose parece ter ampla dispersão geográfica. $\mathrm{O}$ hospedeiro intermediário, a paca, tem larga distribuição, ocorrendo desde o sul do México até o Estado de Santa Catarina no Brasil ${ }^{3}$.

$O$ reconhecimento de doença hidática no homem fora das zonas de distribuição das hidatidoses clássicas, particularmente em áreas tropicais silvestres, deve levantar a possibilidade do diagnóstico de doença hidática policistica. Nessas circunstâncias, deve ser investigado o possível contacto do paciente com cães que tenham ingerido visceras de pacas.

\section{SUMMARY}

Four of the patients with polycystic hydatid disease observed by us reported that they were aware of the presence of liver disease in the pacas hunted for food. The viscera of these animals were usually given to domestic dogs. All of our 7 patients reported contact with dogs that had previously ingested viscera of pacas. Examination of the liver considered to be diseased by one of the patients and removed from $a$ paca killed in the same region (State of Acre, Brazil) from which the human cases originated showed the presence of hydatid cysts. The characteristics of the rostellar hooks of the protoscolex indicated that this was the larval form of Echinococcus vogeli. These observations confirm the participation of pacas in the biological cycle of $\mathrm{E}$. vogeli and the pathway through which man may become an alternative intermediate host of this echinococcus.

Key-words: Echinococcosis. Polycystic hydatid disease. Echinococcus vogeli.

\section{REFERÊNCIAS BIBLIOGRÁFICAS}

1. Brumpt E, Joyeux C. Description d'un nouvel echinocoque: Echinococcus cruzi n.sp. Annales de Parasitologie Humaine et Comparée 2: 226-231, 1924.
2. D'Alessandro A, Rausch RL, Cuello C, Aristizabal N. Echinococcus vogeli in man, with a review of polycystic hydatid disease in Colombia and neighboring countries. The American Journal of Tropical Medicine and Hygiene 28: 303-317, 1979.

3. D'Alessandro A, Rausch RL, Morales GA, Collet S, Angel D. Echinococcus infections in Colombian animals. The American Journal of Tropical Medicine and Hygiene 30: 1263-1276, 1981.

4. Ferreira MS, Rocha A, Gonçalves EG, Carvalho AM, Nishioka SA, Andrade NB. Um caso de hidatidose policística autóctone de Minas Gerais, Brasil. Revista da Sociedade Brasileira de Medicina Tropical 20: 181-186, 1987.

5. Gardner SL, Rausch RL, Camacho OCJ. Echinococcus vogeli. Rausch and Bernstein, 1972, from the paca, Cuniculus paca L. (Rodentia: Dasyproctidae), in the Departamento de Santa Cruz, Bolivia. Joumal of Parasitology 74: 339-402, 1988.

6. Lutz A. Observação de uma cutia infeccionada com Echinococcus. Revista da Sociedade Cientifica de São Paulo 2: 113-114, 1907.

7. Meneghelli UG. Calcificações hepáticas múltiplas decorrentes de doença hidática policistica. Revista Goiana de Medicina 31: 53-60, 1985.

8. Meneghelli UG. Hidatidose na Amazônia brasileira. Archivos Internacionales de la Hidatidosis 29: 79-80, 1989.

9. Meneghelli UG, Barbó MLP, Magro JE, Bellucci AD, Velludo MASL. Polycystic hydatid disease (Echinococcus vogeli): clinical and radiological manifestations and treatment with albendazole of a patient from the Brazilian Amazon region. Arquivos de Gastroenterologia 23: 177-183, 1986.

10. Pacheco PRG, Komma MD, Pinto RNL, Souza LCS, Pereira LIA. Doença hidática policística. Relato de um caso procedente do Pará. Revista da Sociedade Brasileira de Medicina Tropical 19 (supl): 67, 1986.

11. Rausch RL, Bernstein JJ. Echinococcus vogeli sp.n (Cestoda: Taeniidae) from the bush dog, Speothos venaticus (Lund). Zeitschrift für Tropenmedizin und Parasitologie 23: 25-34, 1972.

12. Rausch RL, D'Alessandro A, Ohbayashi M. The taxonomic status of Echinococcus cruzi Brumpt and Joyeux, 1924 (Cestoda: Taeniidae) from an agouti (Rodentia: Dasyproctidae) in Brazil. Journal of Parasitology 70: 295-302, 1984.

13. Rausch RL, Rausch VR, D'Alessandro A. Discrimination of the larval stages of Echinococcus oligarthrus (Diesing, 1863) and $E$, vogeli Rausch and Bernstein, 1972 (Cestoda: Taeniidae), The American Journal of Tropical Medicine and Hygiene 27: 1195-1202, 1978.

14. Timmerman A, Andrade DR, Hutzler RU, Marinho IS, Uliana SRB. Terapêutica da hidatidose hepática com albendazol: relato de 1 caso. In: Resumos do IX Congresso Brasileiro de Hepatologia, resumo 85, São Paulo, 1986. 